\title{
FINITE COVERS OF 3-MANIFOLDS CONTAINING ESSENTIAL TORI
}

\author{
JOHN LUECKE
}

\begin{abstract}
It is shown in this paper that if a Haken 3-manifold contains an incompressible torus that is not boundary-parallel then either it has a finite cover that is a torus-bundle over the circle or it has finite covers with arbitrarily large first Betti number.
\end{abstract}

In [He 4], Hempel conjectures that every Haken 3-manifold has a finite cover whose fundamental group has a nontrivial representation to the integers (i.e. the group is indicable). The conjecture is proved in this paper in the case that the Haken manifold contains an incompressible torus. In particular, it is shown (Theorem 1.1) that if Haken manifold contains an incompressible torus that is not boundaryparallel then either it has a finite cover that is a torus-bundle over the circle or it has finite covers with arbitrarily large first Betti number.

Hempel's conjecture arises in the context of the following question: (1) does every irreducible 3-manifold with infinite fundamental group have a finite cover which is Haken? An affirmative answer to this would be a big step toward the classification of 3-manifolds with infinite fundamental group. How would one detect such a cover? A common way of showing that an irreducible 3-manifold is Haken is to show that its fundamental group has a nontrivial representation to the integers, then use this fact to find a map from the 3-manifold to the circle for which the pre-image of some regular value is an incompressible surface. Thus question (1) leads to: (2) does every irreducible 3-manifold have a finite cover whose fundamental group has a nontrivial representation to the integers? An affirmative answer to (2) would certainly imply one for (1); however, the converse is not known. In fact, the conjecture of Hempel stated in the first paragraph says exactly that (1) implies (2).

For example, let $M$ be the union of two knot complements identified along their boundaries in such a way that the meridian of each knot complement is identified with the longitude of the other. Then $M$ is Haken, but $\pi_{1}(M)$ has no nontrivial representation to the integers $\left(H_{1}(M)=0\right)$. However, it is shown in [He 4] that in many cases $M$ will have a finite cover whose fundamental group does have such a representation. Note that there $M$ contains an incompressible torus that is not boundary parallel, hence Theorem 1.1 applies. In fact, the argument used to prove Theorem 1.1 is a generalization of that used in [He 4] for this example.

This paper is taken from part of the author's Ph.D. dissertation and as such owes much to Cameron Gordon.

Received by the editors February 20, 1986 and, in revised form, September 16, 1987.

1980 Mathematics Subject Classification (1985 Revision). Primary 57M05, 57M10; Secondary $20 \mathrm{E} 07$.

This work was supported in part by NSF grant DMS-8504683. 
The paper is organized as follows. The main theorem (Theorem 1.1) is stated and proved in $\S 1$ assuming Theorems 2.4 and 3.2. Afterwards, at the end of this section, a key, technical lemma is proven that will be used in the proofs of both Theorems 2.4 and 3.2. $\S \S 2$ and 3 are devoted to the proofs of Theorems 2.4 and 3.2 .

0. Terminology. A group is said to be indicable [Hi, p. 241] (or Z-representable [He 4]) if it has a nontrivial representation to the integers. A group is said to be virtually indicable (or virtually $Z$-representable) if it has a subgroup of finite index that has a nontrivial representation to the integers.

$X(M)$ is defined at the beginning of $\S 2$ and is defined only when $M$ is a Seifert fibered space. However, $\chi($ ) will mean the usual Euler characteristic of whatever is in the parenthesis (e.g. a finite graph or a surface).

Let $S$ be a 2 -submanifold in a 3 -manifold, $M$. Then by $U(S)$ we mean an open, regular neighborhood of $S$ in $M$.

Let $S$ be a disjoint collection of two-sided surfaces in a 3-manifold. Let $U(S)$ be an open regular neighborhood of these surfaces in $M$. Then one can associate the dual graph, $\Gamma(S)$, to $S$ by

$$
\begin{aligned}
& \text { Vertices of } \Gamma(S)=\text { components of } M-U(S), \\
& \text { Edges of } \Gamma(S)=\text { components of } S,
\end{aligned}
$$

such that if $e$ is an edge of $\Gamma(S)$ with vertices $v_{1}$ and $v_{2}$, if $S_{e}$ is the component of $S$ corresponding to $e$, and if $M_{1}$ and $M_{2}$ are the components of $M-U(S)$ corresponding to $v_{1}$ and $v_{2}$, then $M_{1}$ and $M_{2}$ are the components of $M-U(S)$ on either side of $U\left(S_{e}\right)$.

FACT 0.1 . Let $M$ be a connected 3-manifold and $S$ be a disjoint collection of two-sided surfaces in $M$. Then $\operatorname{rank}\left(H_{1}(M ; Z)\right) \geq \operatorname{rank} H_{1}(\Gamma(S) ; Z)$.

Let $\Gamma$ be a finite, connected graph.

Define

$$
\begin{aligned}
& e(\Gamma)=\text { number of edges of } \Gamma . \\
& v(\Gamma)=\text { number of vertices of } \Gamma . \\
& \chi(\Gamma)=\text { Euler characteristic of } \Gamma=v(\Gamma)-e(\Gamma) .
\end{aligned}
$$

FACT 0.2 . Let $\Gamma$ be a connected graph. Then $\pi_{1}(\Gamma)$ is the free group on $1-\chi(\Gamma)$ generators. In particular, $H_{1}(\Gamma, Z)$ is the free abelian group of rank $1-\chi(\Gamma)$.

1. Proof of Theorem 1.1. The main purpose of this section is to use the results of $\S \S 2$ and 3 to prove the following.

THEOREM 1.1. Let $M$ be a compact, connected, orientable, irreducible 3manifold containing an incompressible torus that is not boundary parallel. Then either $M$ has a finite cover that fibers as a torus-bundle over the circle or for any positive integer, $k$, there is a finite cover, $\rho_{k}: \tilde{M}_{k} \rightarrow M$, such that $\operatorname{rank}\left(H_{1}\left(\tilde{M}_{k}\right)\right)>k$.

As mentioned in the introduction, Theorem 1.1 verifies a special case of a conjecture made in [He 4] that the fundamental group of a Haken 3-manifold is virtually indicable.

By the techniques given below in the proof of Theorem 1.1, the hypothesis that the incompressible torus is not boundary-parallel can be dropped in the statement 
of Theorem 1.1 (i.e. the existence of the incompressible torus is enough), if one also allows in the conclusion of the theorem the possibility that $M$ is finitely covered by the annulus $\times$ circle.

One can define the first virtual Betti number of a 3-manifold, $M$, to be the supremum over the first Betti number of finite covers of $M$ (see [K-L]). After rephrasing Theorem 1.1 in this terminology it is interesting to compare Theorem 1.1 with the conjecture that the first virtual Betti number of a closed hyperbolic 3 -manifold is infinity.

PROOF OF THEOREM 1.1. Let $M$ be as in the hypothesis of 1.1 .

We make the following definition of a torus decomposition rather than use the more natural, canonical decomposition of Jaco-Shalen-Johannson, because we want to say in the case where $M$ is Seifert fibered that $M$ has a nonempty torus decomposition (or is finitely covered by a torus-bundle over the circle, see claim 1.1.1).

DEFINITION. Let $T$ be a collection of incompressible tori in the interior of an orientable 3-manifold, $N$, such that each component of $N-U(T)$ is either Seifert fibered or has the property that its interior is a hyperbolic manifold. Furthermore, assume that no component of $M-U(T)$ is the annulus $\times$ circle unless there is exactly one component of $M-U(T)$. Then $T$ will be called a torus decomposition of $N$.

Note that the annulus $\times$ circle has only the empty torus decomposition.

ClaIM 1.1.1. Either $M$ is Seifert fibered and has a finite cover which fibers as a torus-bundle over the circle or $M$ has a nonempty torus decomposition, $T=$ $\left\{T_{1}, \ldots, T_{l}\right\}$.

PROOF OF CLAIM. By hypothesis $M$ contains an incompressible torus which is not boundary-parallel. Applying the Jaco-Shalen-Johannson Torus Decomposition Theorem [J, p. 173] and Thurston's Uniformization Theorem [Th 2, pp. 89-91], this means that $M$ either has a nonempty torus decomposition or is Seifert fibered. Theorem 2.5 then finishes the argument. Q.E.D.

Thus for the proof of Theorem 1.1 we assume that $M$ has a nonempty torus decomposition, $T$. We associate the dual graph, $\Gamma(T)$, to the collection of tori, $T$, in $M$ (see $\S 0$ ). Given a positive integer $k$, repeated application of Claim 1.1.2 below implies that either there is a finite cover $\rho_{k}: \tilde{M}_{k} \rightarrow M$ where $\tilde{M}_{k}$ has a torus decomposition, $\tilde{T}_{k}$, such that $\chi\left(\Gamma\left(\tilde{T}_{k}\right)\right)<-k$ or $M$ has a finite cover which is a torus-bundle over the circle. In the former case we then have $\operatorname{rk}\left(H_{1}\left(\tilde{M}_{k}\right)\right)>k$. This proves the theorem.

Thus we are reduced to proving the following.

ClAIM 1.1.2. Let $M$ be a compact, orientable, connected 3-manifold with a nonempty torus decomposition, $T=\left\{T_{1}, \ldots, T_{l}\right\}$. Then there is a finite cover, $p: \tilde{M} \rightarrow M$, such that either

(1) $\tilde{M}$ is a torus-bundle over the circle; or

(2) $\tilde{T}=p^{-1}(T)$ is a torus decomposition of $\tilde{M}$ with $\chi(\Gamma(\tilde{T}))<\chi(\Gamma(T))$.

PROOF OF CLAIM. $M$ has a nonempty torus decomposition, $T=\left\{T_{1}, \ldots, T_{l}\right\}$. Let $\left\{M_{i}\right\}_{i=1, n}$ be the components of $M-U(T)$. We think of $M$ as the union of the pieces $\left\{M_{i}\right\}_{i=1, n}$ whose boundary components are identified via homeomorphisms - which we will refer to as the gluing maps of $M$. 


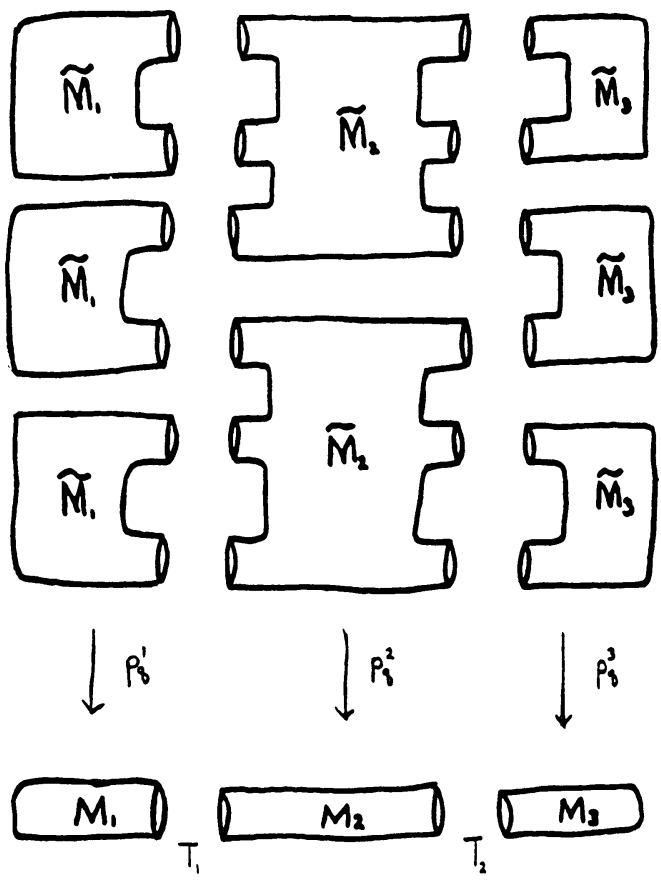

In this figure: $m=6, t_{1}=3, t_{2}=2, t_{3}=3$

FIGURE 1

After passing, possibly, to a finite cover, we may assume that each component, $M_{i}$, of $M-U(T)$ either has a hyperbolic interior or is Seifert fibered over an orientable surface with $X\left(M_{i}\right)<0$ (Theorem 2.6).

We first outline the argument for the claim: For each piece, $M_{i}$, of the decomposition and for all but finitely many primes, $q$, we construct a regular cover (using Theorems 2.4 or 3.2 - according to whether the piece is hyperbolic or Seifert fibered, resp.) such that this cover restricted to each of its toral boundary components is the canonical $q^{2}$-fold cover. This allows us to glue the covers of the pieces together to get (see Figure 1) a covering of $M, \tilde{M} . \tilde{M}$ then has a natural torus decomposition, $\tilde{T}$, coming from $T$. We construct the cover above each piece in the decomposition of $M$ so that each toral boundary component of that piece has at least two components in its preimage. This guarantees that $\chi(\Gamma(\tilde{T}))<\chi(\Gamma(T))$.

We will now give a detailed argument. By applying either Theorem 2.4 or Theorem 3.2 to each piece, $M_{i}$, in the decomposition of $M$ (according to whether the piece is Seifert fibered or hyperbolic, resp.) there is a prime, $q$, such that for every $i=1, n$ there is a finite, connected, regular cover $p_{q}^{i}: \tilde{M}_{i} \rightarrow M_{i}$ where, if $R$ is a component of $\partial M_{i}$, then $\left(p_{q}^{i}\right)^{-1}(R)$ consists of more than one component; furthermore, if $\tilde{R}$ is a component of $\left(p_{q}^{i}\right)^{-1}(R)$, then $\left.p_{q}^{i}\right|_{\tilde{R}}: \tilde{R} \rightarrow R$ is the characteristic $q^{2}$-fold cover. Note that we pick the same $q$ for each $i=1, n$. 
Denote by $\eta_{i}$ the index of $p_{q}^{i}: \tilde{M}_{i} \rightarrow M_{i}$. Then $\left(p_{q}^{i}\right)^{-1}$ (component of $\partial M_{i}$ ) is exactly $\eta_{i} / q^{2}$ copies of a torus (each of which is the $q^{2}$-fold cover under $p_{q}^{i}$ ). Furthermore, $\eta_{i} / q^{2}>1$.

REMARK. Let $T^{\prime}$ be a component of $\partial M_{i}$ and $T^{\prime \prime}$ be a component of $\partial M_{j}$ such that $T^{\prime}$ is identified with $T^{\prime \prime}$ in $M$ by some gluing homeomorphism, $g: T^{\prime} \rightarrow T^{\prime \prime}$ (i.e. $T^{\prime}$ and $T^{\prime \prime}$ correspond to the same component of $T$ ). Let $\tilde{T}^{\prime}, \tilde{T}^{\prime \prime}$ be components of $\left(p_{q}^{i}\right)^{-1}\left(T^{\prime}\right),\left(p_{q}^{j}\right)^{-1}\left(T^{\prime \prime}\right)$ (respectively). Because $\left.p_{q}^{i}\right|_{\tilde{T}^{\prime}}: \tilde{T}^{\prime} \rightarrow T^{\prime}$ and $\left.p_{q}^{j}\right|_{\tilde{T}^{\prime \prime}}: \tilde{T}^{\prime \prime} \rightarrow$ $T^{\prime \prime}$ are both the characteristic $q^{2}$-fold covers, there is a homeomorphism, $\tilde{g}: \tilde{T}^{\prime} \rightarrow$ $\tilde{T}^{\prime \prime}$, that covers $g: T^{\prime} \rightarrow T^{\prime \prime}$.

Let $m=1$.c. m. $\left(\eta_{1} / q^{2}, \eta_{2} / q^{2}, \ldots, \eta_{n} / q^{2}\right)$. Take $t_{i}=m /\left(\eta_{i} / q^{2}\right)$ copies of $\tilde{M}_{i}$ $(i=1, n)$. See Figure 1 . The preceding remark allows us to glue the components of $\bigsqcup_{i=1, n}\left(\amalg_{j=1, t_{i}} \tilde{M}_{i}\right)$ together, via lifts of the gluing maps of $M$, to obtain a connected, finite cover, $p: \tilde{M} \rightarrow M$. This is the desired cover.

Let $\tilde{T}=p^{-1}(T)$. We must show that $\chi(\Gamma(\tilde{T}))<\chi(\Gamma(T))$. Since for each component, $T_{i}$, of $T$ there are exactly $m$ components in $p^{-1}\left(T_{i}\right)$, it follows that $e(\Gamma(\tilde{T}))=m \cdot e(\Gamma(T))$. Similarly, for each component $M_{i}(i=1, n)$ of $M-U(T)$ there are exactly $t_{i}$ components in $p^{-1}\left(M_{i}\right)$, thus

$$
v(\Gamma(\tilde{T}))=\sum_{i=1, n} t_{i}
$$

Now $t_{i} \leq m / 2$ for every $i=1, n$. So

$$
v(\Gamma(\tilde{T})) \leq n \cdot m / 2=v(\Gamma(T)) \cdot m / 2 .
$$

Thus

$$
\begin{aligned}
\chi(\Gamma(\tilde{T})) & =v(\Gamma(\tilde{T}))-e(\Gamma(\tilde{T})) \leq \frac{m}{2} \cdot v(\Gamma(T))-m \cdot e(\Gamma(T)) \\
& =\frac{m}{2} \cdot \chi(\Gamma(T))-\frac{m}{2} \cdot e(\Gamma(T))=\frac{m}{2} \cdot(\chi(\Gamma(T))-e(\Gamma(T))) .
\end{aligned}
$$

Since $\Gamma(T)$ is connected, $\chi(\Gamma(T)) \leq 1$. Since $e(\Gamma(T)) \geq 1, \chi(\Gamma(T))-e(\Gamma(T)) \leq 0$. Also, recall that $1 \leq t_{i} \leq m / 2$. Thus we have

$$
\chi(\Gamma(\tilde{T})) \leq \chi(\Gamma(T))-e(\Gamma(T))<\chi(\Gamma(T)) .
$$

This finishes the proof of Claim 1.1.2 and the proof of Theorem 1.1. Q.E.D.

$\S \S 2$ and 3 are devoted essentially to the proofs of Theorems 2.4 and 3.2 (respectively), which are used in Theorem 1.1, above. The following lemma is the key to producing the necessary covers over each of the components of $M-U(T)(M$ and $T$ as in the proof of Theorem 1.1) and will be used in both $\S \S 2$ and 3. I would like to thank Cameron Gordon for pointing out to me the lemma and its proof. The argument is a generalization of that used in [He 4].

Recall that $S L_{2}(F)$ is the group of all $2 \times 2$ matrices with determinant 1 over the field $F$, and $P S L_{2}(F)$ is the quotient group of $S L_{2}(F)$ by its center: i.e.

$$
P S L_{2}(F)=S L_{2}(F) /\left\langle\left(\begin{array}{cc}
-1 & 0 \\
0 & -1
\end{array}\right)\right\rangle \text {. }
$$

If $g \in P S L_{2}(F)$, define $\operatorname{tr}(g)$ to be the trace of some lift of $g$ to $S L_{2}(F)$-note this is defined only to a factor of \pm 1 . 
LEMMA 1.4. Let $G$ be a finitely generated group and $\tau: G \rightarrow P S L_{2}(\mathbf{C})$ be a faithful representation of $G$. Let $\lambda_{1}, \ldots, \lambda_{m} \in G$ be such that $\lambda_{i} \neq 1_{G}$ and $\operatorname{tr}\left(\tau\left(\lambda_{i}\right)\right)= \pm 2,1 \leq i \leq m$. Let $y \in G$ satisfy $\operatorname{tr}(\tau(y)) \neq \pm 2$. Then for all but finitely many primes, $q$, there is a finite field, $F_{q}$, of characteristic $q$ and a representation $\tau_{q}: G \rightarrow P S L_{2}\left(F_{q}\right)$ such that

(1) if $g \in G$ and $\operatorname{tr}(\tau(g))= \pm 2$, then $\tau_{q}(g)$ has order 1 or $q$ in $P S L_{2}\left(F_{q}\right)$. In particular, $\tau_{q}\left(\lambda_{i}\right)$ has order $q$ in $P S L_{2}\left(F_{q}\right)$.

(2) $\operatorname{tr}\left(\tau_{q}(y)\right) \not \equiv \pm 2$ in $F_{q}$.

PROOF. Since $\operatorname{tr}\left(\tau\left(\lambda_{i}\right)\right)=2$ and $\tau\left(\lambda_{i}\right)$ is not the identity, $\tau\left(\lambda_{i}\right)$ has at least one nonzero entry off the diagonal. For each $i=1, m$ set $\eta_{i} \in \mathbb{C}$ to be one such entry in $\tau\left(\lambda_{i}\right)$. (So that $\eta_{i} \in \mathbb{C}$, pick some lift of $\tau\left(\lambda_{i}\right)$ to $S L_{2}(\mathbb{C})$.) Let $A$ be the ring generated by

\{the entries of the matrix, $\tau(g)$, for every $g \in G\}$

$$
\cup\left\{\eta_{i}, \eta_{i}^{-1}\right\}_{i=1, m} \cup Z \cup\left\{(\operatorname{tr}(\tau(y))-2)^{-1},(\operatorname{tr}(\tau(y))+2)^{-1}\right\} .
$$

$A$ is finitely generated as a ring since $G$ is finitely generated. Therefore the group of units of $A$ is finitely generated as a group (see $[\mathbf{S}]$ or $[\mathbf{A - S}$, p. 7]). This implies that only a finite number of primes can be units in $A$. For every prime, $q$, which is not a unit in $A$ there is a maximal ideal $M_{q} \subset A$ with $q \in M_{q}$. Then $F_{q}=A / M_{q}$ is a field which is finitely generated as a ring over $Z_{q}$. Consequently, $F_{q}$ is a finite field of characteristic $q$ [A-M, p. 84].

So for all but finitely many primes, $q$, we get the induced representation, $\tau_{q}: G \rightarrow$ $P S L_{2}\left(F_{q}\right)$. One easily verifies that if $g \in G$ such that $\operatorname{tr}(\tau(g))= \pm 2$ then $\tau_{q}(g)$ has order 1 or $q$ (for example, use the fact that $\tau(g)$ is conjugate to $\left.\left[\begin{array}{ll}1 & 1 \\ 0 & 1\end{array}\right]\right)$. In the particular case when $g=\lambda_{i}$, for some $i=1, n$, the fact that $\eta_{i}$ is a unit in $A$ implies that $\bar{\eta}_{i} \neq 0$ in $F_{q}$; hence, $\tau_{q}\left(\lambda_{i}\right)$ is of order $q$.

To prove $(2)$, note that $(\operatorname{tr}(\tau(y))-2),(\operatorname{tr}(\tau(y))+2)$ are invertible in $A$ so $\overline{\operatorname{tr}(\tau(y))-2}, \overline{\operatorname{tr}(\tau(y))+2} \not \equiv 0$ in $F_{q}$. Thus $\operatorname{tr}\left(\tau_{q}(y)\right) \not \equiv \pm 2$ in $F_{q}$. Q.E.D.

2. The Seifert fibered pieces. For our notation on Seifert fibered spaces see [He 1, pp. 115 118].

DEFInition. Let $\eta: S \rightarrow F$ be a Seifert fibered space with orbit surface $F$ and exceptional fibers of type $\left\{\left(n_{i}, m_{i}\right)\right\}_{i=1, t}$. Define

$$
X(S)=\chi(F)-\sum_{i=1}^{t}\left(1-\frac{1}{n_{i}}\right)
$$

where $\chi(F)=$ Euler characteristic of $F$.

Note. $X(S)$ is the Euler characteristic of the orbifold structure on $F$ induced from the map $\eta: S \rightarrow F$, where the singular points of the orbifold structure come from the singular fibers of $S$ under the projection $\eta$ (see [Th 1, Chapter 13]).

If $S$ is an orientable Seifert fibered space with orientable orbit surface, $F, \partial F \neq$ $\varnothing$, and projection map, $\eta: S \rightarrow F$, then $\pi_{1}(S)$ has the following presentation 
[He 1, pp. 116-117]:

(I) $\pi_{1}(S)=\left\{\begin{array}{l}t, d_{1}, \ldots, d_{k}, c_{1}, \ldots, c_{u}, \\ a_{1}, b_{1}, \ldots, a_{g}, b_{g}\end{array}\right.$

$$
\left.\begin{array}{l}
{\left[a_{i}, t\right]=\left[b_{i}, t\right]=1, i=1, g ;} \\
{\left[d_{i}, t\right]=1, i=1, k ;} \\
{\left[c_{i}, t\right]=1, i=1, u ;} \\
c_{i}^{n_{i}}=t^{s_{i}}, i=1, u ; \\
d_{1} \cdots d_{k} c_{1} \cdots c_{u} \prod_{i=1}^{g}\left[a_{i}, b_{i}\right]=1
\end{array}\right\}
$$

where $t$ represents the fiber; $\left\{a_{i}, b_{i}\right\}_{i=1, g},\left\{c_{i}\right\}_{i=1, u},\left\{d_{i}\right\}_{i=1, k}$ represent the generators of a section, $\mathscr{F}$, of the circle-bundle $\eta^{-1}(F-$ disk neighborhoods of singular points). In particular, the $\left\{d_{i}\right\}_{i=1, k}(k>0)$ represent the components of $\mathscr{F} \cap \partial S$, the $\left\{c_{i}\right\}_{i=1, u}$ represent the components of $\mathscr{F} \cap \eta^{-1}$ (disk neighborhoods of the singular points), and $F$ has genus $g$.

Since $t$ is central in $\pi_{1}(S)$, we may talk about $\pi_{1}(S) /\langle t\rangle$ and we have

$$
\frac{\pi_{1}(S)}{\langle t\rangle}=\left\{\begin{array}{ll}
a_{1}, b_{1}, \ldots, a_{g}, b_{g}, & c_{i}^{n_{i}}=1, i=1, u, \\
d_{1}, \ldots, d_{k}, c_{1}, \ldots, c_{u} & d_{1} d_{2} \cdots d_{k} c_{1} \cdots c_{u} \prod_{i=1}^{g}\left[a_{i}, b_{i}\right]=1
\end{array}\right\} .
$$

Recall that we can classify the elements of $P S L_{2}(C)$ as hyperbolic, elliptic or parabolic by thinking of $P S L_{2}(C)$ as the orientation preserving isometries of $H^{3}$ where

(a) $g \in P S L_{2}(C)$ is elliptic if it fixes an arc in $H^{3}(\operatorname{tr}(g) \neq \pm 2)$,

(b) $g \in P S L_{2}(C)$ is parabolic if it has no fixed point in $H^{3}$ and has exactly one fixed point on the sphere at $\infty$ (in this case $\operatorname{tr}(g)= \pm 2$ ),

(c) $g \in P S L_{2}(C)$ is hyperbolic otherwise, i.e. it has no fixed points in $H^{3}$ and exactly two fixed points in the sphere at $\infty$ (in this case $\operatorname{tr}(g) \neq \pm 2$ ).

THEOREM 2.1 (FRICKE). Let $S$ be an orientable Seifert fibered space, $\eta: S \rightarrow$ $F$, where $F$ is an orientable surface with nonempty boundary and where $X(S)<0$. With $\pi_{1}(S)$ presented as in (I) there is a discrete, faithful representation $\tau: \pi_{1}(S) /\langle t\rangle$ $\rightarrow P S L_{2}(C)$ such that

(a) $\tau\left(a_{i}\right), \tau\left(b_{i}\right)$ are hyperbolic, $i=1, g$,

(b) $\tau\left(c_{i}\right)$ is elliptic, $i=1, u$,

(c) $\tau\left(d_{i}\right)$ is parabolic, $i=1, k$.

PROOF. See [M, pp. 98-99].

LEMma 2.2. Let $S$ and $\tau: \pi_{1}(S) /\langle t\rangle \rightarrow P S L_{2}(C)$ be as in Theorem 2.1. Pick $y \in \pi_{1}(S) /\langle t\rangle$ such that $\tau(y)$ is hyperbolic. Then for all but finitely many primes, $q$, there is a representation, $\tau_{q}: \pi_{1}(S) /\langle t\rangle \rightarrow P S L_{2}\left(F_{q}\right)$, where $F_{q}$ is a finite field of characteristic $q$, such that

(1) if $d_{i}, i=1, k$, is as in the presentation (II) of $\pi_{1}(S) /\langle t\rangle$, then $\tau_{q}\left(d_{i}\right)$ has order $q$ in $P S L_{2}\left(F_{q}\right)$; and

(2) $\operatorname{tr}\left(\tau_{q}(y)\right) \not \equiv \pm 2$ in $F_{q}$.

PROOF. This is an immediate consequence of Theorem 2.1 and Lemma 1.4.

LEMMA 2.3. Let $S$ be an orientable Seifert fibered space, $\eta: S \rightarrow F$, where $F$ is an orientable surface with nonempty boundary. For all but finitely many primes, 
$q$, there is a map $f_{q}: \pi_{1}(S) \rightarrow Z_{q}$ such that $f_{q}(t) \neq 0$ (when $t$ represents the fiber of $S$ ).

ProOF. $t$ is of infinite order in $H_{1}(S)$.

THEOREM 2.4. Let $S$ be a connected orientable Seifert fibered space, $\eta: S \rightarrow F$, where $F$ is an orientable surface with nonempty boundary. Assume $X(S)<0$. Then for all but finitely many primes, $q$, there is a finite, connected, regular cover, $p_{q}: \tilde{S}_{q} \rightarrow S$, such that if $T$ is a component of $\partial S$ and $\tilde{T}$ is any component of $p_{q}^{-1}(T)$, then $\left.p_{q}\right|_{\tilde{T}}: \tilde{T} \rightarrow T$ is the characteristic $q^{2}$-fold cover. Furthermore, $p_{q}^{-1}(T)$ consists of more than one component.

ProOF. Let $S$ be as in the theorem and (I) be a presentation of $\pi_{1}(S)$. Let $q$ be a prime for which the maps, $\tau_{q}: \pi_{1}(S) /\langle t\rangle \rightarrow P S L_{2}\left(F_{q}\right)$ (Lemma 2.2) and $f_{q}: \pi_{1}(S) \rightarrow Z_{q}$ (Lemma 2.3) are defined. Note that $q$ can be any but a finite number of primes. Let $\bar{\tau}_{q}: \pi_{1}(S) \rightarrow P S L_{2}\left(F_{q}\right)$ be the homomorphism obtained by composing $\tau_{q}$ with the quotient map $\pi_{1}(S) \rightarrow \pi_{1}(S) /\langle t\rangle$. Let $h_{q}=\bar{\tau}_{q} \times f_{q}: \pi_{1}(S) \rightarrow$ $P S L_{2}\left(F_{q}\right) \times Z_{q}$.

Let $p_{q}: \tilde{S}_{q} \rightarrow S$ be the connected, regular cover corresponding to $\operatorname{ker}\left(h_{q}\right)$. If $T$ is a component of $\partial S$ then $\pi_{1}(T)=\left\langle d_{i}, t\right\rangle$ for some $i=1, k$. By construction of $h_{q}$ we have that $\operatorname{ker}\left(h_{q} \mid\left\langle d_{i}, t\right\rangle\right)=\left\langle d_{i}^{q}, t^{q}\right\rangle$. Thus if $\tilde{T}$ is a component of $p_{q}^{-1}(T)$, $\left.p_{q}\right|_{\tilde{T}}: \tilde{T} \rightarrow T$ must be the characteristic $q^{2}$-fold cover. Let $y \in \pi_{1}(S) /\langle t\rangle$ be as in Lemma 2.2. Since $\operatorname{tr}\left(\tau_{q}(y)\right) \not \equiv \pm 2$ in $F_{q}, h_{q}(y) \notin h_{q}\left(\left\langle d_{i}, t\right\rangle\right) \forall i=1, k$. Thus every component of $\partial S$ has at least two components in its preimage. Q.E.D. (Theorem 2.4)

The following two theorems are also used in the proof of Theorem 1.1.

THEOREM 2.5. Let $M$ be a connected, irreducible 3-manifold which is also a Seifert fibered space. Assume that $M$ contains an embedded, incompressible torus that is not boundary parallel. Then either

(a) $M$ has a finite cover that fibers as a torus-bundle over the circle; or

(b) $M$ has a nonempty torus decomposition.

ProOF. Let $T$ be an embedded, incompressible torus in $M$. Since $M$ is irreducible, we can isotop $T$ until it is either vertical (i.e. a union of regular fibers) or horizontal (i.e. transverse to the fibers) [Wa, p. 319]. Let $\eta: M \rightarrow F$ be the Seifert projection of $M$ onto its orbit surface, $F$. If $T$ is horizontal, then $\eta \mid T: T \rightarrow F$ is a branched covering of $F$ in which the singular points of the covering arise from the points of $T$ where $T$ intersects the singular fibers of $M . \eta \mid T$ can now be used to construct a circle-bundle over the torus that covers $M$ by pulling back the Seifert fibration over $F$. For example see Theorem 1.2 of $[\mathbf{G a}]$. The circle-bundle over the torus can then be rewritten as a torus-bundle over the circle.

So we may assume that $T$ is vertical. In this case, each component of $M-$ $U(T)$ is Seifert fibered. Since $T$ is not boundary parallel, no component of $M-$ $U(T)$ is the circle $\times$ annulus - unless here also $M$ fibers as a torus-bundle over the circle. Q.E.D.

Theorem 2.4 applies to Seifert fibered spaces, $\eta: S \rightarrow F$, where $F$ is orientable and $X(S)<0$. The following theorem is used in the proof of Theorem 1.1 to allow us to make that assumption. 
THEOREM 2.6. Let $M$ be a connected Haken 3-manifold. Let $T=\left\{T_{1}, \ldots, T_{l}\right\}$ be a nonempty torus decomposition of $M$. Then there is a finite cover, $p: \tilde{M} \rightarrow M$, such that either

(1) $\tilde{M}$ is a torus-bundle over the circle; or

(2) $\tilde{M}$ has a nonempty torus decomposition, $\tilde{T}$, such that each component, $\tilde{M}_{i}$, of $\tilde{M}-U(\tilde{T})$ either has a hyperbolic interior or is Seifert fibered over an orientable orbit surface with $X\left(\tilde{M}_{i}\right)<0$.

ProOF. Let $M, T$ be as in the theorem.

ClaIM. If $M_{i}$ is a component of $M-U(T)$ which is a Seifert fibered space then either $X\left(M_{i}\right)<0$ or $M_{i}$ is homeomorphic to the orientable circle-bundle over the annulus or Moebius band.

PROOF OF CLAIM. Let $M_{i}$ be a component of $M-U(T)$ and assume that $X\left(M_{i}\right) \geq 0$. Then, since $M_{i}$ is bounded, $M_{i}$ must be Seifert fibered over

(i) the disk with 0 or 1 singular fibers;

(ii) the disk with exactly two singular fibers, each of type $(2,1)$;

(iii) the Moebius band with no singular fibers;

(iv) the annulus with no singular fibers.

Case (i) cannot hold since $\partial M_{i}$ is incompressible in $M_{i}$. In case (ii) we note that the Seifert fibered space over the disk with two singular fibers of type $(2,1)$ is homeomorphic to the circle bundle over the Moebius band [Wa, p. 323]. Q.E.D. (Claim)

Each component, $M_{i}$, of $M-U(T)$ is either hyperbolic or Seifert fibered; furthermore, if $M_{i}$ is Seifert fibered and $X\left(M_{i}\right) \geq 0$ then $M_{i}$ is homeomorphic as a Seifert fibered space to the orientable circle-bundle over the annulus or Moebius band (this requires refibering when $M_{i}$ is as in (ii) of the claim).

If some component, $M_{i}$, of $M-U(T)$ is a Seifert fibered space over a nonorientable orbit surface, then $M_{i}$ has a double cover corresponding to the orientation double cover of its orbit surface. Furthermore, the components of $\partial M_{i}$ lift to this cover. By taking one copy of this double cover for each component of $M-U(T)$ that is Seifert fibered over a nonorientable surface, taking two copies of each component otherwise, and identifying these along their torus boundaries via the identification between the components of $M-U(T)$ (since the boundary components of each component of $M-U(T)$ lift, so do the gluing maps), we obtain a double cover, $p: \tilde{M} \rightarrow M$. This cover has the property that if $\tilde{T}=p^{-1}(T)$ then each component of $\tilde{M}-U(\tilde{T})$ is either Seifert fibered over an orientable surface or hyperbolic.

Thus there is a finite cover, $p: \tilde{M} \rightarrow M$ (equal to $M$ or the double cover above), and a system of incompressible tori, $\tilde{T}$, in $\operatorname{int}(\tilde{M})$ such that each component, $\tilde{M}_{i}$, of $\tilde{M}-U(\tilde{T})$ is either hyperbolic, Seifert fibered over an orientable orbit surface with $X\left(\tilde{M}_{i}\right)<0$, or the orientable circle-bundle over the annulus.

Let $\tilde{M}_{i}$ be a component of $\tilde{M}-U(\tilde{T})$ that is the orientable circle-bundle over the annulus - that is, $\tilde{M}_{i} \approx S^{1} \times$ annulus $\approx$ torus $\times$ interval. We may reduce the number of components of $\tilde{T}$ by amalgamating the components of $\partial \tilde{M}_{i}$ via $\tilde{M}_{i} \approx$ torus $\times$ interval, unless the two components of $\partial \tilde{M}_{i}$ represent the same componcnt of $\tilde{T}$, or unless $\tilde{M}_{i}=\tilde{M}$. Via this simplification of $\tilde{T}$, we have that either $\tilde{M}$ is the torus-bundle over the circle, $\tilde{M}$ is the annulus $\times$ circle, or no component of $\tilde{M}-U(\tilde{T})$ is the orientable circle-bundle over the annulus. If $\tilde{M}$ is the annulus 
$\times$ circle then each component of $\tilde{M}-U(\tilde{T})$ must be the annulus $\times$ circle. By construction of $\tilde{M}$, each component of $M-U(T)$ must be either the annulus $\times$ circle or the orientable circle-bundle over the Moebius band. In this case $M$ must be either the annulus $\times$ circle or the orientable circle-bundle over the Moebius band. But neither has a nonempty torus decomposition. Q.E.D. (2.6)

3. The hyperbolic pieces. Let $N$ be a compact manifold such $\operatorname{int}(N)$ is a hyperbolic manifold. There is a discrete, faithful representation, $\tau: \pi_{1}(N) \rightarrow$ $P S L_{2}(C)$. If $T^{\prime}$ is a torus component of $\partial N$, and $T^{\prime}$ is incompressible in $N$, then the discreteness of $\tau$ implies that $\tau\left(\pi_{1}\left(T^{\prime}\right)\right)$ consists of parabolic elements of $P S L_{2}(C)$.

Let $\left\{T_{j}\right\}_{j=1, k}$ be a collection of toral components of $\partial N$, each of which is incompressible in $N$. Let $i_{j}: T_{j} \rightarrow N$ be the inclusion. Remember, $\left(i_{j}\right)_{*}: \pi_{1}\left(T_{j}\right) \rightarrow \pi_{1}(N)$ is an injection.

LEMMA 3.1. Let $N$ be a compact, orientable 3-manifold and $\left\{T_{j}\right\}_{j=1, k}$ be a collection of toral components of $\partial N$. For every $j=1, k$, there is a basis $\left\{\mu_{j}, \lambda_{j}\right\}$ of $\pi_{1}\left(T_{j}\right)$ such that for all but finitely many primes, $q$, there is a map, $h_{q}: \pi_{1}(N) \rightarrow$ $\bigoplus_{\text {rank } H_{1}(N)} Z_{q}$, with the property that $\operatorname{ker}\left(h_{q} \circ\left(i_{j}\right)_{*}\right)=\left\langle\mu_{j}^{q}, \lambda_{j}^{q}\right\rangle$ or $\left\langle\mu_{j}^{q}, \lambda_{j}\right\rangle$ for every $j=1, k$.

PROOF. Let $h: \pi_{1}(N) \rightarrow \bigoplus_{\text {rank } H_{1}(N)} Z$ be the composition of the Hurewicz map with the quotient map $H_{1}(N) \rightarrow H_{1}(N) / \operatorname{Tor}\left(H_{1}(N)\right)$. Let $i_{j}: T_{j} \rightarrow N$ be the inclusion map. Then the rank of $\operatorname{ker}\left(h \circ\left(i_{j}\right)_{*}\right)$ is 0 or 1 . Let $\left\{\mu_{j}, \lambda_{j}\right\}, j=1, k$, be a basis of $\pi_{1}\left(T_{j}\right)$ such that if the rank $\left(\operatorname{ker}\left(h \circ\left(i_{j}\right)_{*}\right)\right)=1$ then $\operatorname{ker}\left(h \circ\left(i_{j}\right)_{*}\right)=\left\langle\lambda_{j}\right\rangle$ (otherwise $\left\{\mu_{j}, \lambda_{j}\right\}$ can be any basis).

For every prime, $q$, define $h_{q}: \pi_{1}(N) \rightarrow \bigoplus_{\text {rank } H_{1}(N)} Z_{q}$ by composing $h$ with the quotient homomorphism $\bigoplus Z \rightarrow \bigoplus Z /(q \cdot(\bigoplus Z))$. Then $\left\{\mu_{j}, \lambda_{j}\right\}, j=1, k$, and $h_{q}$ are as desired. Q.E.D.

THEOREM 3.2. Let $N$ be a compact 3-manifold whose interior is a hyperbolic manifold. Assume that $N$ is not the torus $\times$ interval. Let $\left\{T_{i}\right\}_{i=1, k} \subset \partial N$ be a nonempty collection of incompressible tori. For all but finitely many primes, $q$, there is a finite, connected, regular cover, $p_{q}: \tilde{N}_{q} \rightarrow N$, such that

(1) if $\tilde{T}_{i}$ is a component of $p_{q}^{-1}\left(T_{i}\right)$, for some $i=1, k$, then $\left.p_{q}\right|_{\tilde{T}_{i}}: \tilde{T}_{i} \rightarrow T_{i}$ is the characteristic $q^{2}$-fold cover; and

(2) $p_{q}^{-1}\left(T_{i}\right)$ contains more than one component for every $i=1, k$.

PROOF. As in the proof of Theorem 2.4, this theorem will follow from the claim below if we let $p_{q}: \tilde{N}_{q} \rightarrow N$ be the cover corresponding to $\operatorname{ker}\left(f_{q}\right)$ [parts (1) and (2) of the theorem will follow from (1) and (2) (respectively) of the claim].

ClaIM. For all but finitely many primes, $q$, there is a homomorphism, $f_{q}: \pi_{1}(N) \rightarrow G_{q}$, where $G_{q}$ is a finite group and where

(1) $\operatorname{ker}\left(\left.f_{q}\right|_{i_{*}\left(\pi_{1}\left(T_{j}\right)\right)}\right)$ is the characteristic subgroup of index $q^{2}$ for every $j=1, k$; and

(2) there is a $y \in \pi_{1}(N)$ such that, for every $j=1, k, f_{q}(y) \notin f_{q}\left(i_{*}\left(\pi_{1}\left(T_{j}\right)\right)\right)$.

PROOF OF CLAIM. As mentioned earlier, there is a discrete, faithful representation, $\tau: \pi_{1}(N) \rightarrow P S L_{2}(C)$ such that if $t \in i_{*}\left(\pi_{1}\left(T_{j}\right)\right)$ for some $j=1, k$, then $\tau(t)$ is parabolic. Furthermore, as $N$ is not the torus $\times$ interval, there is a $y \in \pi_{1}(N)$ such that $\tau(y)$ is hyperbolic. 
Let $\left\{\mu_{j}, \lambda_{j}\right\}$ be the basis of $\pi_{1}\left(T_{j}\right)$ described in Lemma 3.1. Applying Lemma 1.4 to $\tau$, along with the elements $\left\{\left(i_{j}\right)_{*}\left(\lambda_{j}\right)\right\}_{j=1, k}$, we obtain for all but finitely many primes, $q$, a representation $\tau_{q}: \pi_{1}(N) \rightarrow P S L_{2}\left(F_{q}\right)$ where $F_{q}$ is a finite field of characteristic $q$ and

(a) if $t \in \pi_{1}\left(T_{j}\right)$, for some $j=1, k$, then $\tau_{q}\left(\left(i_{j}\right)_{*}(t)\right)$ has order 1 or $q$ in $P S L_{2}\left(F_{q}\right)$. In particular, $\tau_{q}\left(\left(i_{j}\right)_{*}\left(\lambda_{j}\right)\right)$ has order $q$ in $P S L_{2}\left(F_{q}\right)$ for every $j=1, k$; and

(b) $\operatorname{tr}\left(\tau_{q}(y)\right) \not \equiv \pm 2$ in $F_{q}$.

Let $q$ be any prime for which $\tau_{q}: \pi_{1}(N) \rightarrow P S L_{2}\left(F_{q}\right)$ is defined and for which $h_{q}: \pi_{1}(N) \rightarrow \bigoplus_{\text {rank } H_{1}(N)} Z_{q}$ is as in Lemma 3.1 . (Note: This excludes only finitely many primes.)

Define $f_{q}=h_{q} \times \tau_{q}: \pi_{1}(N) \rightarrow\left(\bigoplus Z_{q}\right) \times P S L_{2}\left(F_{q}\right) . f_{q}$ satisfies conditions (1) and (2) of the claim: Condition (2) follows from the fact that if $t \in \pi_{1}\left(T_{j}\right)$, for some $j=1, k$, then $\operatorname{tr}\left(\tau_{q}\left(\left(i_{j}\right)_{*}(t)\right)\right) \equiv \pm 2$ (in $\left.F_{q}\right)$, yet $\operatorname{tr}\left(\tau_{q}(y)\right) \not \equiv \pm 2$ (in $F_{q}$ ). Condition (1) says that $\operatorname{ker}\left(f_{q} \circ\left(i_{j}\right)_{*}\right)=\left\langle\mu_{j}^{q}, \lambda_{j}^{q}\right\rangle$ for every $j=i, k$. This follows quickly from the constructions of $h_{q}$ and $f_{q}$. Q.E.D. (3.2)

\section{BIBLIOGRAPHY}

[A-M] M. F. Atiyah and I. G. McDonald, Introduction to commutative algebra, Addison-Wesley, 1969.

[A-S] Roger C. Alperin and Peter B. Shalen, Linear groups of finite cohomological dimension, preprint.

[Ga] David Gabai, On 3-manifolds finitely covered by surface-bundles, Low-dimensional Topology and Kleinian Groups, London Math. Soc. Lecture Notes, no. 112, Cambridge Univ. Press, Cambridge, England, 1986.

[He1] John Hempel, 3-manifolds, Ann. of Math. Studies, no. 86, Princeton Univ. Press, Princeton, N.J., 1976.

[He2] _ Homology of coverings, Pacific J. Math. 112 (1984), 83-114.

[He3] _ Orientation reversing involutions and the first Betti number for finite coverings of 3manifolds, Invent. Math. 67 (1982), 133-143.

[He4] _ Virtually Haken manifolds, preprint.

[Hi] Graham Higman, The units of group rings, Proc. London Math. Soc. 46 (1940), 231-248.

[J] William Jaco, Lectures on 3-manifold topology, CBMS Regional Conf. Ser. in Math., 43, Amer. Math. Soc., Providence, R.I., 1979.

[K-L] S. Kojima and D. D. Long, Virtual Betti numbers of some hyperbolic 3-manifolds, preprint.

[M] Wilhelm Magnus, Noneuclidean tesselations and their groups, Academic Press, New York, 1974.

[S] P. Samuel, À propos du théorème des unités, Bull. Sci. Math. (2) 90 (1966), 89-96.

[Th1] William Thurston, The geometry and topology of 3-manifolds, lecture notes.

[Th2] _ , Three dimensional manifolds, Kleinian groups, and hyperbolic geometry, Proc. Sympos. Pure Math., vol 39, Amer. Math. Soc., Providence, R.I., 1983, pp. 87-111.

[Wa] F. Waldhausen, Eine Klasse von 3-dimensionalen Mannigfaltigkeiten I, Invent. Math. 3 (1967), 308-333; II, Invent. Math. 4 (1967), 87-117. 